\title{
Protocolos Multibanda para Descoberta de Vizinhança em Redes Ad Hoc de Ondas Milimétricas
}

\author{
Davi da Silva Brilhante ${ }^{1}$, José Ferreira de Rezende ${ }^{1}$ \\ ${ }^{1}$ Laboratório de Modelagem, Análise e Desenvolvimento \\ de Redes e Sistemas de Computação (LAND) \\ Programa de Engenharia de Sistemas e Computação (PESC) \\ Universidade Federal do Rio de Janeiro (UFRJ) \\ Rio de Janeiro - RJ - Brazil
}

\begin{abstract}
Propagation at millimeter waves band, i.e. up from $30 \mathrm{GHz}$, is very susceptible to path loss attenuation, which can be mitigated using highly directional antennas. Hence, the usage of this band in ad hoc networks increases the complexity of neighbor discovery since the knowledge of neighbors' physical location becomes essential to proceed with communication. To face these challenges, multiband protocols have been proposed, which uses an omnidirectional control channel in a different band from millimeter waves data channel. Due to unstable characteristics of millimeter waves channel, the control channel is used to transmit information concerning neighbor discovery. The two protocols proposed in this work rely on this feature and aim to build a global knowledge about nodes' location as well as to maintain it in the case of nodes mobility or if some obstacle arises. Both protocols are compared with another protocol found in the literature in terms of delay on the neighbor discovery, decreasing the time for total discovery up to $75 \%$.
\end{abstract}

Resumo. A propagação na banda de ondas milimétricas, ou seja, a partir de $30 \mathrm{GHz}$, é altamente suscetível à atenuação por perda de percurso. Esta acentuada atenuação é mitigada pelo uso de antenas altamente direcionais. Assim, o uso dessa banda em redes ad hoc aumenta a complexidade na descoberta de vizinhos, pois se torna essencial obter a localização física dos vizinhos para proceder com a comunicação. Para lidar com esse desafio, protocolos multibanda foram propostos na literatura, os quais fazem uso de um canal de controle omnidirecional em uma banda diferente do canal de dados em ondas milimétricas. Devido às instabilidades deste último canal, o canal de controle é usado para transmissão das informações pertinentes à realização de busca dos vizinhos. $O s$ dois protocolos propostos neste trabalho fazem uso dessa funcionalidade e visam construir o conhecimento global do posicionamento dos nós, assim como manter esta informação em caso de mudanças de posicionamento ou surgimento de obstáculos. Ambos os protocolos são comparados com um outro protocolo da literatura em termos da latência na descoberta de vizinhança, diminuindo em até $75 \%$ o tempo para descoberta total da rede.

\section{Introdução}

A evolução dos dispositivos móveis tem aumentado a demanda por conexões de rede sem fio com larguras de banda cada vez maiores. No entanto, as bandas não licenciadas já 
disponíveis tornaram-se superpopuladas, aumentando as interferências co-canal e de canal adjacente. Isto resulta na limitação das taxas de transferência, impactando a experiência dos usuários dessas redes e comprometendo aplicações multimídia, como vídeo em alta definição, gaming em realidade virtual, entre outros.

Com o objetivo de utilizar uma faixa de frequência mais livre de interferências e que suporta uma maior largura de banda, agências reguladoras liberaram uma faixa do espectro de frequências em torno de $60 \mathrm{GHz}$ para uso não-licenciado. A banda de frequências que se inicia em $30 \mathrm{GHz}$ e termina em $300 \mathrm{GHz}$ é chamada de ondas milimétricas (millimiter waves, $\mathrm{mm} W$ ), contendo a faixa em questão de $60 \mathrm{GHz}$.

Essa faixa de frequências, no entanto, impõe diversos desafios. A perda de propagação experimentada pelo sinal é muito severa em relação às bandas anteriormente utilizadas [Niu et al. 2015]. Então, faz-se necessário o uso de antenas direcionais, as quais aumentam o ganho em uma certa direção, dentro de uma determinada abertura (beamwidth) e com isso há o aumento do alcance dos sinais propagados por antenas deste tipo. Por outro lado, o comprimento de onda da ordem de milímetros permite a utilização de antenas com tamanho bastante reduzido, o que torna possível o uso de múltiplas antenas, proporcionando feixes com abertura cada vez menor e consequentemente com maior ganho [Korakis et al. 2008].

Em redes que fazem uso de antenas direcionais, para uma transmissão ser bem sucedida, é necessário que, ao efetuá-la, os nós estejam direcionados um para o outro. Para isso, os nós da rede devem conhecer a localização relativa dos demais nós, mais especificamente, que conheçam os ângulos de chegada (Angle of Arrival, AoA) das transmissões dos demais nós. Isso requer um mecanismo denominado de descoberta de vizinhança (Neighbor Discovery), o qual determina a cada instante tais ângulos de chegada.

No entanto, os mecanismos de descoberta de vizinhança precisam ser eficientes para permitir a rápida inicialização da rede, pois a demora na obtenção das informações dos ângulos de chegada inviabiliza a ocorrência de transmissões [Ning et al. 2011]. A intermitência característica do canal e a suscetibilidade dos sinais em ondas milimétricas até a completa interrupção dos enlaces agravam a necessidade de descobertas para a recuperação rápida dessas informações de localização a fim de que a rede não experimente longos retardos.

O processo de descoberta de vizinhança pode ser realizado através do beamforming par a par. O padrão IEEE 802.11ad define uma técnica de descoberta de vizinhança, o Sector Sweeping (SSW). No SSW, o nó iniciador transmite em cada feixe da sua antena direcional um quadro sonda, enquanto o nó respondedor recebe esses quadros sonda de forma quasi omnidirecional. O SSW ocorre ainda no sentido oposto, com o respondedor enviando suas sondas em modo quasi omni, enquanto o iniciador transita entre seus feixes [Nitsche et al. 2014].

Com o objetivo de aumentar a eficiência do padrão IEEE 802.11ad e também mitigar os efeitos da surdez, os autores em [Sim et al. 2016] utilizam o IEEE 802.11ac, na banda de $5 \mathrm{GHz}$, para trocar mensagens de controle em modo omnidirecional. Essa abordagem, denominada multibanda, proporcionou um aumento de $65 \%$ na taxa de transferência total da rede em relação ao IEEE 802.11ad. A descoberta de vizinhança foi também abordada usando uma perspectiva multibanda em [Park et al. 2015]. O trabalho 
propõe um protocolo centralizado baseado em SSW usando a banda de controle no modo omnidirecional, denominada doravante de banda omnidirecional. As mensagens de controle estabelecem as capacidades dos nós da rede e do canal, tais como quais dispositivos são capazes de operar na banda de ondas milimétricas, o número de feixes disponíveis e a modulação a ser utilizada. O beamforming e as transmissões ocorrem sob a reserva da banda em ondas milimétricas. No entanto, o overhead causado pelas mensagens de controle podem impactar diretamente no desempenho deste protocolo se escalado para descoberta total de uma rede com um certo número de dispositivos.

Neste trabalho, propomos dois protocolos de descoberta de vizinhança centrados em um nó líder fazendo uso do paradigma multibanda. Em um dos protocolos, o nó líder determina o ângulo de chegada de todos os nós da rede do seu ponto de vista e em seguida, com tais informações, o nó líder constrói e transmite um mapa da rede no canal de controle em uma outra banda. O Outro protocolo escuta informações de modo oportunístico a respeito da localização e responde a essas informações. Transmitindo essas mensagens de controle em broadcast em modo omnidirecional, a rede torna-se robusta à surdez e às falhas na entrega das mensagens de controle. De posse do mapa da rede e da informação de localização do nó líder, cada nó pode estimar a direção para qualquer outro nó, adquirindo um conhecimento global da rede. Esses protocolos diminuíram o tempo levado para a descoberta total da rede em cerca de $75 \%$ em uma rede com 25 nós.

O trabalho segue organizado da seguinte forma: nas seções 2 e 3, apresentamos os protocolos propostos, MuNDi MaPP (Multiband Neighbor Discovery Map Propagation based Protocol) e Go MuNDi (Gossip Multiband Neighbor Discovery), respectivamente; na seção 4, conduzimos análises matemáticas sobre a probabilidade de erro na localização de um nó e modelamos matematicamente o tempo de permanência na banda de controle; os resultados obtidos são apresentados na seção 5; e na seção 6 trazemos as conclusões e as perspectivas futuras.

\section{MuNDi MaPP}

O protocolo MuNDi MaPP foi concebido com o objetivo de diminuir o tempo levado para que os nós realizem beamforming e as informações de localização sejam mantidas de forma centralizada ao invés de distribuída. A manutenção centralizada visa corrigir os erros detectados e distribuir mensagens de correção através de um único nó, diminuindo assim o tempo de ocupação das bandas de controle ou de dados, no caso de um beamforming precisar ser refeito.

Considere uma rede sem fio composta por $K$ nós equipados com interfaces operando em duas bandas, uma em ondas milimétricas e outra em uma banda de controle. No presente trabalho, iremos adotar como banda de controle a banda WiFi não licenciada em $2.4 \mathrm{GHz}$, conforme o padrão definido pelo IEEE 802.11. Por se tratar de um cenário indoor os nós não contam com GPS ou qualquer outro dispositivo que gere informação de localização.

Dentre os $K$ nós, um deles será eleito para atuar como líder da rede e desempenhará funções de controle e gerenciamento. Os demais $K-1$ nós, denominados aqui de nós regulares, irão se associar ao nó líder.

Consideramos que os nós formam uma rede completa, ou seja, todos os nós são alcançáveis com apenas um salto, em ambas as bandas de operação da rede. Iremos 
considerar que o líder já foi estabelecido previamente, segundo um critério de eleição como, por exemplo, o nó mais próximo ao centróide da rede.

Os nós regulares se associarão ao líder na banda de controle ao ouvirem as mensagens de HELLO, transmitidas periodicamente. Após a associação dos nós regulares, há uma etapa de distribuição de senhas, que contabilizam de 1 a $K-1$ os nós da rede. O líder distribui as senhas em broadcast, no seguinte formato $\left[\left(i d_{1}, s_{1}\right),\left(i d_{2}, s_{2}\right), \ldots,\left(i d_{k-1}, s_{k-1}\right)\right]$, onde $i d_{i}$ é um identificador único de um nó e $s_{i}$ a senha que o nó recebeu. A função das senhas é escalonar o processo de beamforming, que tem duração fixa. Cada nó ocupará uma janela de beamforming, segundo a senha recebida. Os nós que não estão realizando beamforming permanecem em estado ocioso.

Após realizado o processo de beamforming, o líder manterá uma tabela contendo os ângulos ou feixes usados para se comunicar com os nós regulares, a largura de feixe utilizada no beamforming e as distâncias estimadas para alcançá-los. A estimativa da distância é um processo factível em ondas milimétricas devido ao comportamento quase óptico das ondas eletromagnéticas nessa faixa de frequências. Em [Palacios et al. 2017] e [Olivier et al. 2016], são testados algoritmos para estimar a distância relativa entre nós e o mapeamento de ambientes utilizando ondas milimétricas. Os resultados obtidos alcançam até $50 \mathrm{~cm}$ de precisão em mais de $80 \%$ dos casos.

As entradas na tabela armazenadas pelo líder seguem o seguinte formato $\left[\alpha_{i}, d_{i}, C_{i}\right]$, onde $i=1,2, \ldots, K-1 . \quad \alpha_{i}$ representa o ângulo encontrado pelo líder ao realizar beamforming com o $i$-ésimo nó. Caso o ângulo encontrado não seja preciso o suficiente, o líder armazenará o feixe $B_{i}$, ao invés do ângulo $\alpha_{i}$. A distância estimada pelo líder através do processo de beamforming é representada por $d_{i}$. $C_{i}$ representa a capacidade dos nós, expressa em quantidade de feixes que o nó $i$ utilizou para realizar beamforming com o líder. Detalhes do protocolo são mostrados na Figura 1.

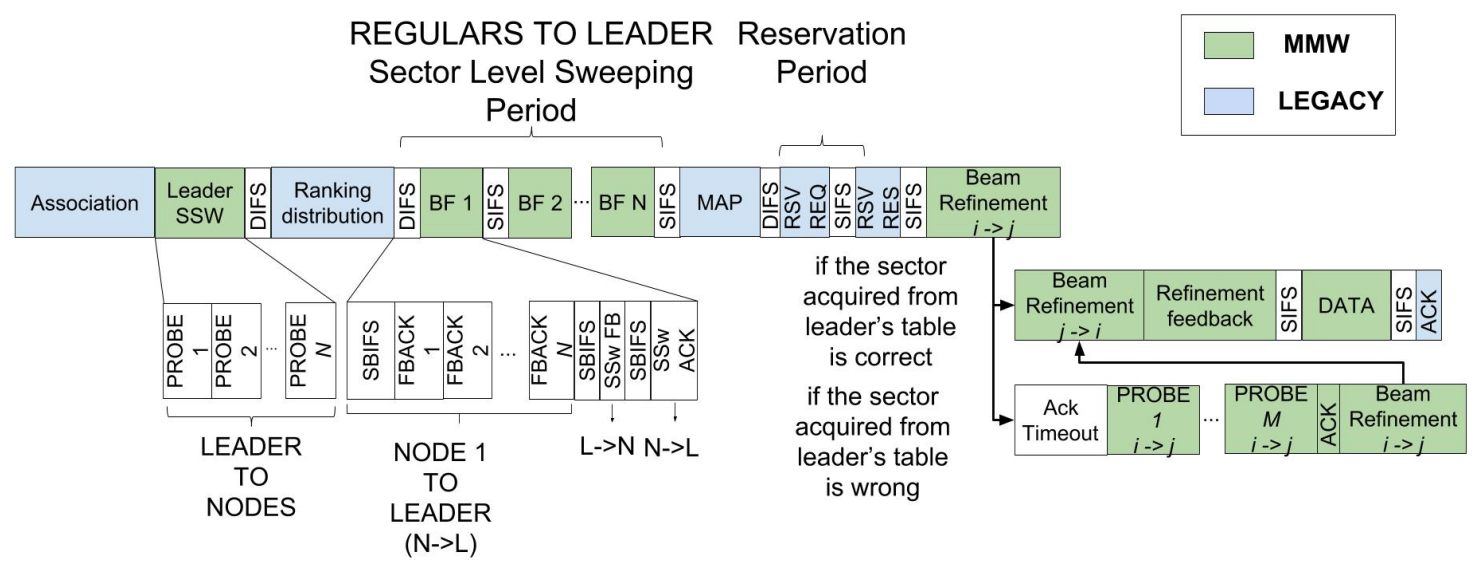

Figura 1. Diagrama do tempo do Protocolo MuNDi MaPP

Realizado o processo de beamforming, o líder irá transmitir o mapa da rede em broadcast na banda de controle. Este mapa consiste em uma tupla $\left[\alpha_{i}, d_{i}\right]$ contendo o ângulo e a distância estimada para cada nó, relativos ao líder. A partir dessas informações, qualquer nó pode calcular o ângulo de transmissão ou recepção para qualquer outro nó, conforme passamos a explicar.

O esquema proposto adiante não restringe o sistema à existência de um norte 
comum para os nós, ou seja, uma direção para a qual todos os nós estão orientados. A seguir, demonstraremos como realizar os cálculos com ou sem norte. Considere um nó $i$ transmissor e um nó $j$ receptor, estes nós formam um ângulo $\alpha_{i j}$ com o líder, como pode ser visto na Figura 2. Os nós $i$ e $j$ estão a uma distância $d_{i}$ e $d_{j}$ do líder, respectivamente. Cada nó regular recebeu essas informações na tupla enviada pelo líder, uma vez que $\alpha_{i j}=$ $\alpha_{i}-\alpha j$ é o ângulo diferença entre $i$ e $j$. Assim o ângulo $\theta_{i j}$ necessário para $i$ comunicar-se com $j$ é dado por:

$$
\theta_{i j}=\arctan \left(\frac{d_{j} \operatorname{sen}\left(\alpha_{i j}\right)}{d_{i}-d_{j} \cos \left(\alpha_{i j}\right)}\right)
$$

No entanto, $\theta_{i j}$ é relativo ao líder e não ao norte do nó $i$, seja este norte comum a todos os nós ou não. Cada nó regular estabelecerá um ângulo $\theta_{i l}$ relativo ao líder para corrigir o ângulo $\theta_{i j}$ obtido. Havendo um norte comum, $\theta_{i l}$ pode ser calculado através da expressão:

$$
\theta_{i l}= \begin{cases}\alpha_{i}-\pi, & \text { se } \theta_{i}>\pi \\ \alpha_{i}+\pi, & \text { caso contrário }\end{cases}
$$

Não havendo um norte em comum entre os nós, o ângulo $\theta_{i l}$ será estabelecido através do processo de beamforming do nó $i$ para o nó líder. Assim, na ausência de um norte comum entre os nós, o beamforming deverá ser realizado nas duas direções, do nó líder para o nó $i$ e vice-versa.

Então o ângulo $\theta_{i j}$ pode ser corrigido para:

$$
\theta_{i j}= \begin{cases}\arctan \left(\frac{d_{j} \operatorname{sen}\left(\alpha_{i j}\right)}{d_{i}-d_{j} \cos \left(\alpha_{i j}\right)}\right)+\theta_{i l}, & \text { se } \alpha_{i}>\alpha_{j} \\ \theta_{i l}-\arctan \left(\frac{d_{j} \operatorname{sen}\left(\alpha_{i j}\right)}{d_{i}-d_{j} \cos \left(\alpha_{i j}\right)}\right), & \text { caso contrário }\end{cases}
$$

Terminada a fase de divulgação do mapa, os nós ficam livres para realizar transmissões.

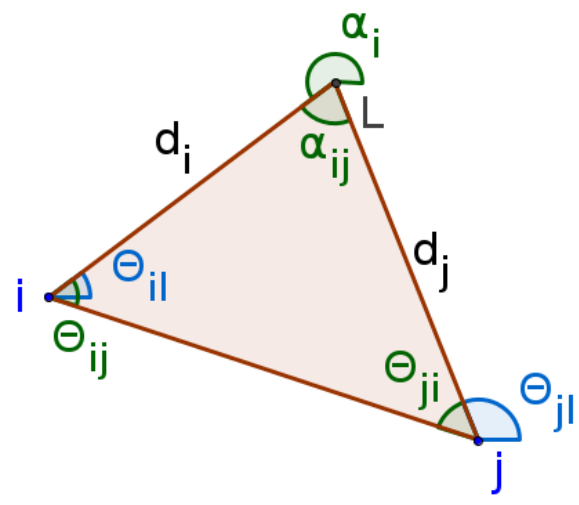

Figura 2. Disposição dos ângulos e distâncias do -math-protocolo MuNDi MaPP

As transmissões ocorrem apenas sob reserva do canal de ondas milimétricas. Um nó que pretende transmitir envia na banda de controle a mensagem RSV REQ, (Reservation Request). A transmissão será iniciada somente quando o nó receptor responder à mensagem de RSV REQ, com uma mensagem do tipo RSV RES (Reservation Response).

A troca de mensagens $R S V R E Q$ e $R S V R E S$ garante que o meio de transmissão na banda de ondas milimétricas esteja livre para troca de mensagens. O nó líder também utiliza essas mensagens para certificar-se que o meio está livre e assim realizar manutenção 
das informações de ângulo e distância. Uma consulta aos nós, na banda de ondas milimétricas, pode verificar se o ângulo que o líder guarda em sua tabela ainda é válido. Caso o ângulo não seja válido, é possível realizar beamforming com o nó em questão.

Outra funcionalidade proveniente desta troca de mensagens, contudo não abordada neste trabalho, é a possibilidade de reuso espacial. Mediante a avaliação das mensagens $R S V R E Q, R S V R E S$ e do mapa, mais de um par de nós pode transmitir simultaneamente na banda de ondas milimétricas, desde que espacialmente não haja interferência decorrente das transmissões simultâneas.

Por fim, já na banda de ondas milimétricas, podem executar o refinamento do beamforming, trocando quadros que levam padrões de treinamento das antenas direcionais. Com o refinamento, a vazão alcançada pode ser maior em relação às técnicas que utilizam apenas troca de quadros. Caso um quadro seja perdido por causa de eventuais imprecisões no cálculo do ângulo, o nó transmissor inicia uma busca ao receptor de forma localizada. Essa busca prioriza os feixes adjacentes ao feixe obtido nos cálculos, uma vez que a maioria dos erros ocorre em feixes vizinhos, como será descrito na subseção 5.1.

\section{Go MuNDi}

O protocolo Go MuNDi (Gossip Multiband Neighbor Discovery) aplica o mesmo princípio de utilizar uma banda omnidirecional para transmissão das mensagens de controle. No entanto, na etapa de realização de beamforming, os nós podem permanecer ativos como receptores na banda de ondas milimétricas mesmo fora da janela reservada para si. Assim, esses nós podem 'ouvir' as mensagens trocadas entre os nós que estão realizando beamforming e o líder. Essas mensagens obtidas oportunisticamente revelam a localização dos vizinhos.

Para executar a escuta oportunística das mensagens durante o Sector Sweeping, os nós devem seguir o funcionamento do líder. Adotando a mesma configuração do líder os nós oportunistas estarão se comportando como se fizessem parte do beamforming, tornando-os aptos para receber essas mensagens da mesma forma que líder as receberá. Se o líder continuar no modo direcional, os nós regulares devem permanecer com as antenas no modo direcional também, a fim de descobrir qual feixe na recepção resultou em um sinal com maior potência. Do contrário, permanecendo o líder em modo quasi omnidirecional, os nós encontrarão qual feixe do vizinho a realizar beamforming gera maior potência recebida.

Finalizada a etapa de beamforming, os nós divulgam mensagens de feedback a respeito dos beamformings que puderam ser ouvidos. Essa etapa ocorre na banda de controle, em modo omnidirecional. Os feedbacks são transmitidos utilizando CSMA-CA. Tomemos dois nós $i$ e $j$ como exemplo. Considere que $i$ capturou os pacotes de beamforming transmitidos por $j$ e o contrário também é verdadeiro. O feedback transmitido por $i$ reporta qual feixe de $j$ ao transmitir durante o beamforming resulta em maior potência recebida por $i$. O nó $j$ age de modo análogo, reportando a $i$, qual feixe gerou a maior potência recebida. Considerando que o canal é recíproco, $i$ e $j$ terão conhecimento dos feixes para transmitir e receber em ambas as direções.

Portanto, considerando uma rede com $K$ nós, excetuando o líder, no caso em que todos os nós da rede escutam todos os seus vizinhos realizando beamforming com o líder, 
cada nó transmitirá $K-1$ mensagens. Ou seja, serão transmitidos $K *(K-1)$ quadros de feedback em um cenário favorável a todos os nós escutarem as transmissões dos seus vizinhos. Caso contrário, os nós que não foram capazes de capturar uma mensagem de SSW deverá executar um beamforming posteriormente com o vizinhos não detectados. Para aumentar a probabilidade de os nós serem ouvidos de forma oportunística por seus vizinhos durante o beamforming, a recepção será realizada sempre em modo quasi omnidirecional. Ao permanecer em modo quasi omnidirecional, o ganho da antena diminui em relação ao modo direcional.

Após a etapa de feedbacks, dá-se início ao período de transmissões. Assim como a proposta anterior, as transmissões ocorrem mediante a reserva prévia do canal, com troca de mensagens entre o transmissor e o receptor. Para alcançar maiores taxas de transmissão, há também a oportunidade de os nós realizarem refinamento do beamforming antes de transmitirem os dados. O diagrama de tempo do protocolo está mostrado na Figura 3. Assim como no protocolo MuNDi MaPP, o protocolo Go MuNDi também distribui senhas para escalonar a realização dos beamformings entre o líder e os nós regulares. Durante o período de transmissão de feedback os nós disputam pelo meio em CSMA-CA notificando seus vizinhos da descoberta oportunística outrora realizada. Findada a fase de entrega dos feedbacks os nós estão livres para transmitir, sob reserva de canal utilizando as mensagens $R S V R E Q$ e RSV RES, realizando ou não o refinamento do beamforming.

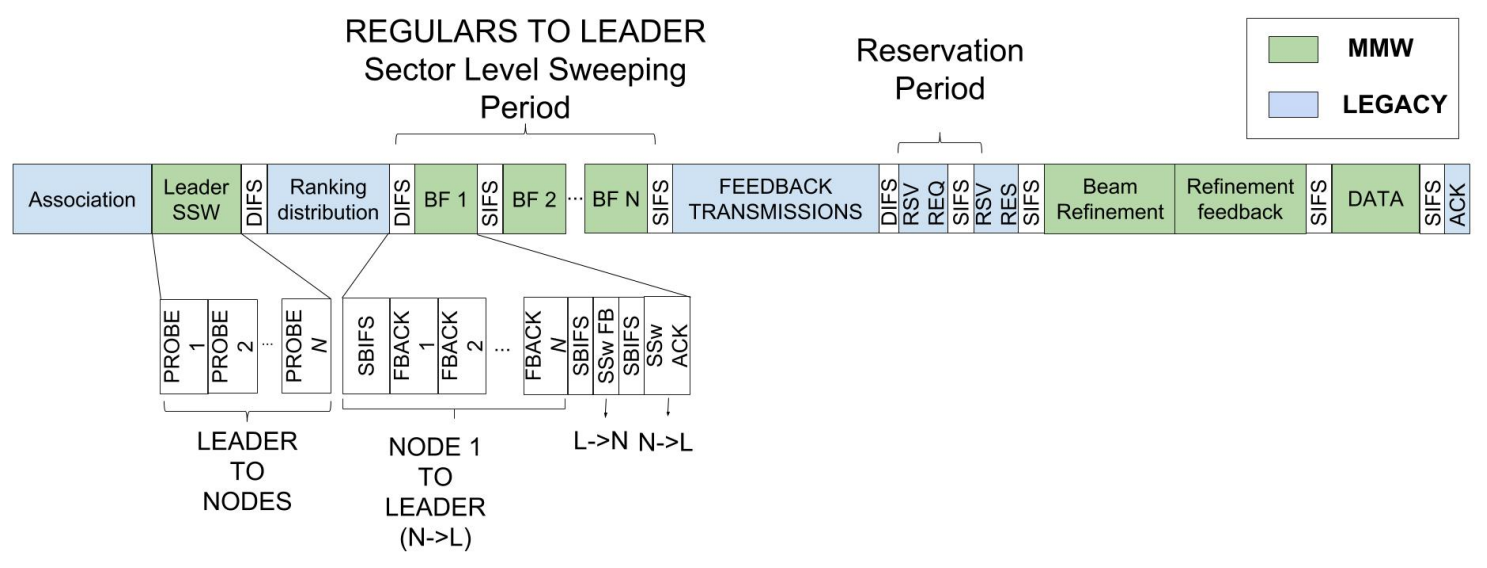

Figura 3. Diagrama do tempo do Protocolo Go MuNDi

\section{Análise Matemática}

Nesta Seção, iremos analisar matematicamente alguns aspectos dos protocolos propostos. O objetivo dessas análises é estabelecer estimativas do tempo esperado para que os protocolos desempenhem algumas de suas funções.

\subsection{Probabilidade de Erro na Estimativa do Feixe}

O protocolo definido na seção 2 é dependente da medida do ângulo e da distância. No entanto, os nós não são capazes de realizar a aquisição dessas informações sem que haja um erro associado. Em um cenário ideal, a equação (1) modela o sistema de forma determinística. Considerando a condição de erro nas medições, modelamos o sistema através de uma variável aleatória contínua $D_{i}$, cujo evento aleatório é a medida da distância do 
líder para o nó $i$. Assim, a equação (1) que determina o ângulo de transmissão do nó $i$ para o nó $j$ pode ser escrita por:

$$
\begin{aligned}
\frac{1}{T} & =\frac{D_{j} \operatorname{sen}\left(\alpha_{i j}\right)}{D_{i}-D_{j} \cos \left(\alpha_{i j}\right)} \\
T & =\frac{\frac{D_{i}}{D_{j}}}{\operatorname{sen}\left(\alpha_{i j}\right)}-\operatorname{cotan}\left(\alpha_{i j}\right)
\end{aligned}
$$

$T$ é uma variável aleatória contínua cuja distribuição de probabilidade ainda é desconhecida. Esta variável aleatória $T$ expressa o valor da cotangente do ângulo $\theta_{i j}$, isto é, o ângulo que o nó $i$ deve utilizar ao transmitir para $j$. Assumindo que $D_{i}$ e $D_{j}$ são variáveis aleatórias com função de densidade de probabilidade $f_{D}(d)$ conforme a seguinte distribuição uniforme:

$$
f_{D}(d)= \begin{cases}\frac{1}{2 \varepsilon} & , \text { se } \hat{d}-\varepsilon \hat{d} \leq d \leq \hat{d}+\varepsilon \hat{d} \\ 0 & , \text { caso contrário }\end{cases}
$$

Onde, $\hat{d}$ é a medida de distância real e sem erro entre o líder e um nó. A constante $\varepsilon$ representa o erro presente na medida da distância realizada pelo líder durante o beamforming. Para facilitar a notação e melhorar a compreensão, nós denotaremos a equação (4) da seguinte maneira:

$$
T=\frac{Z}{\operatorname{sen}\left(\alpha_{i j}\right)}-\operatorname{cotan}\left(\alpha_{i j}\right)
$$

Onde $Z$ é o quociente das variáveis aleatórias $D_{i}$ e $D_{j}$, independentes. Devido a $\operatorname{sen}\left(\alpha_{i j}\right)$ e contan $\left(\alpha_{i j}\right)$ serem constantes, se tratando de uma mudança de escala e uma translação, respectivamente, e sendo ambas operações lineares, podemos tratar de forma matematicamente simples a transformação da variável aleatória $Z$ na variável aleatória $T$.

As funções de distribuição de probabilidade e de distribuição cumulativa do quociente de duas variáveis aleatórias com distribuição uniforme foram demonstradas em [Sakamoto 1943]. Portanto, a variável aleatória $Z$ possui a seguinte distribuição:

$$
f_{Z}(z)= \begin{cases}0 & \text { se } z \leq \frac{\hat{d}_{i}-\varepsilon_{1} \hat{d}_{i}}{\hat{d}_{j}+\varepsilon_{2} \hat{d}_{j}} \\ \frac{1}{8 \hat{d}_{i} \hat{d}_{j} \varepsilon_{1} \varepsilon_{2}}\left[\left(\hat{d}_{j}+\varepsilon_{2} \hat{d}_{j}\right)^{2}-\frac{\left(\hat{d}_{i}-\varepsilon_{1} \hat{d}_{i}\right)^{2}}{z^{2}}\right] & \text { se } \frac{\hat{d}_{i}-\varepsilon_{1} \hat{d}_{i}}{\hat{d}_{j}+\varepsilon_{2} \hat{d}_{j}} \leq z \leq \frac{\hat{d}_{i}}{\hat{d}_{j}} \\ \frac{1}{8 \hat{d}_{i} \hat{d}_{j} \varepsilon_{1} \varepsilon_{2}}\left[\frac{\left(\hat{d}_{i}+\varepsilon_{1} \hat{d}_{i}\right)^{2}}{z^{2}}-\left(\hat{d}_{j}-\varepsilon_{2} \hat{d}_{j}\right)^{2}\right] & \text { se } \frac{\hat{d}_{i}}{\hat{d}_{j}} \leq z \leq \frac{\hat{d}_{i}+\varepsilon_{1} \hat{d}_{i}}{\hat{d}_{j}-\varepsilon_{2} \hat{d}_{j}} \\ 0 & \text { se } z \geq \frac{\hat{d}_{i}+\varepsilon_{1} \hat{d}_{i}}{\hat{d}_{j}-\varepsilon_{2} \hat{d}_{j}}\end{cases}
$$

Como dito anteriormente, por se tratar de uma transformação linear, a transformação da variável $Z$ em $T$ se dará da seguinte forma:

$$
f_{T}(t)=f_{Z}(z)\left|\frac{d z}{d t}\right|
$$

então, $f_{T}(t)$ é dada por:

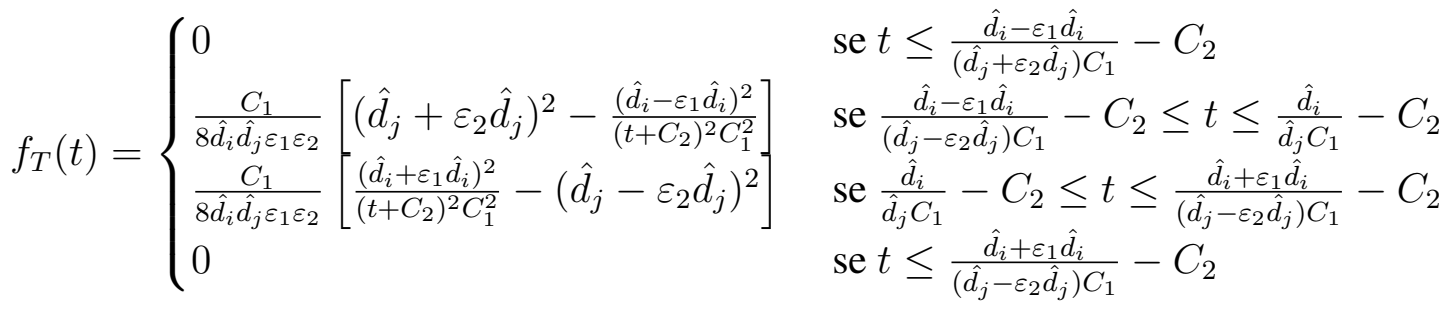


Onde,

$$
\begin{aligned}
& C_{1}=\operatorname{sen}\left(\alpha_{i j}\right) \\
& C_{2}=\operatorname{cotan}\left(\alpha_{i j}\right)
\end{aligned}
$$

\subsection{Análise do Tempo Médio de Transmissão do 802.11}

O objetivo desta análise é estabelecer o tempo esperado para os $K$ nós da rede transmitirem suas mensagens feedback. Como a etapa de feedback é realizada na banda de controle, que opera com CSMA-CA, o período de uma transmissão é não determinístico. O tempo esperado para que um dispositivo STA realize uma transmissão bem sucedida no padrão IEEE 802.11 foi estudado analiticamente em diversos trabalhos. No entanto, optamos por utilizar o modelo descrito pela eq. 10 [Cardoso and de Rezende 2012]. Este modelo permite a inclusão do backoff exponencial no cálculo do tempo médio esperado.

$$
\begin{aligned}
T X_{\text {time }} & =D I F S+\sum_{i=0}^{r} \text { backoff }(i)+(r+1) \times(\text { SIFS } \\
& +A C K+\text { header }+(n * 8 / b)) \\
\text { backoff }(i) & =\frac{1}{2^{i} C W_{\text {min }}}
\end{aligned}
$$

Onde $b$ é a taxa de bits da camada física, $r$ é o número de tentativas que um nó realiza até que conclua uma transmissão e $n$ é o tamanho do payload. backof $f(i)$ assume o valor do tempo médio de backoff para a $i$-ésima tentativa de transmitir e $C W_{\text {min }}$ é o tamanho inicial da janela de contenção. DIFS, SIFS e $A C K$ são, respectivamente, os tempos de DIFS, SIFS e da transmissão de um ACK no padrão utilizado. O termo header representa o tempo para transmissão dos cabeçalhos das camadas física e de enlace.

O número de tentativas de transmissões $r$, deve ser estabelecido de tal forma a transmitir $K * K-1$ mensagens de feedback, como o cenário descrito na Seção 3. Este cenário possui aspecto transitório, uma vez que um nó transmitiu os $K-1$ quadros de feedback, este nó cessa a disputa pelo canal, diminuindo o número de nós disputando o meio. Isto irá se repetir até que não haja mais nenhum nó a transmitir.

A probabilidade de um nó inserido em uma rede com $k$ nós transmitir é dada por:

$$
P_{t x}=\frac{k \tau(1-\tau)^{k-1}}{1-(1-\tau)^{k}}
$$

Onde $\tau$ é a duração média do período de backoff [An et al. 2011]. Podemos então definir o operador $\tau^{x}$, onde $x$ é o número tentativas de transmissão sem sucesso, ou seja, o número de vezes que a janela de contenção sofreu aumento.

$$
\tau^{x}=\frac{2}{2^{x} w+1}
$$

Onde $w$ é o tamanho mínimo da janela de contenção. A probabilidade de ocorrerem $s$ transmissões até que haja uma transmissão bem sucedida pode ser definida por:

$$
\begin{aligned}
P_{s} & =\left[1-\frac{n \tau^{1}\left(1-\tau^{1}\right)^{k-1}}{1-\left(1-\tau^{1}\right)^{k}}\right]\left[1-\frac{n \tau^{2}\left(1-\tau^{2}\right)^{k-1}}{1-\left(1-\tau^{2}\right)^{k}}\right] \\
& \ldots\left[1-\frac{n \tau^{s-1}\left(1-\tau^{s-1}\right)^{k-1}}{1-\left(1-\tau^{s-1}\right)^{k}}\right]\left[\frac{n \tau^{s}\left(1-\tau^{s}\right)^{k-1}}{1-\left(1-\tau^{s}\right)^{k}}\right]
\end{aligned}
$$


Podemos então definir o número esperado de retransmissões $E[r]$ somando as probabilidades de transmitir até que as $m$ retransmissões permitidas pela rede sejam alcançadas.

$$
E[r]=\sum_{s=1}^{m}\left[\prod_{k=1}^{s-1} 1-\frac{n \tau^{k}\left(1-\tau^{k}\right)^{n-1}}{1-\left(1-\tau^{k}\right)^{n}}\right]\left[n \frac{\tau^{s}\left(1-\tau^{s}\right)^{n-1}}{1-\left(1-\tau^{s}\right)^{n}}\right] s
$$

O valor de $r$ para ser substituído na eq. 10 pode ser obtido através de

$$
r=\lceil E[r]\rceil
$$

Onde $\lceil$.$\rceil representa o arredondamento, para o inteiro maior mais próximo (ceiling).$ Os resultados obtidos através da eq. 10 foram comparados com simulações realizadas no software de simulação $N S$-3. O resultado obtido pode ser visto na Figura 4 e os parâmetros da simulação estão descritos na Tabela 1.

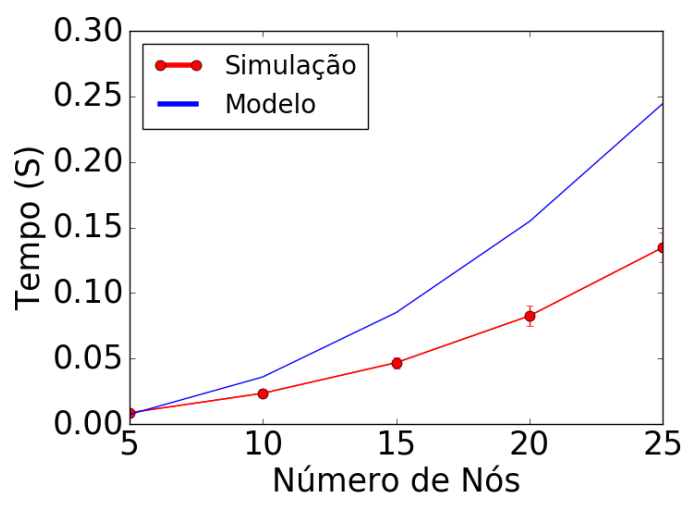

(a)

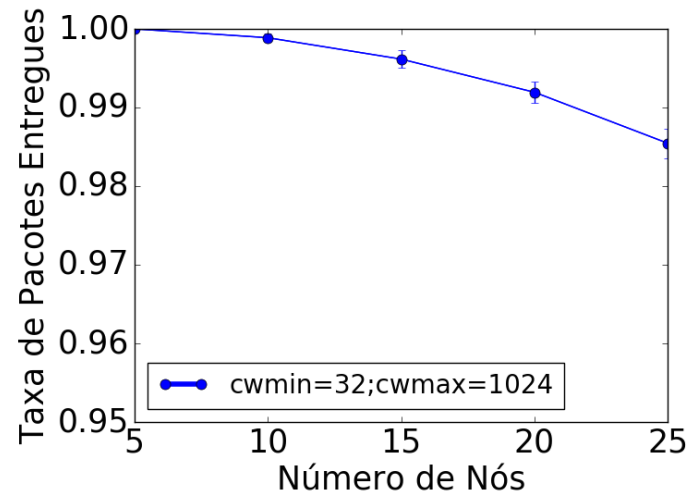

(b)

Figura 4. (a) Comparação entre os resultados da simulação e do modelo proposto; (b) Porcentagem de pacotes entregues com Janela de Contenção igual a 1024

Com base na Figura 4(a) podemos adotar o modelo analítico proposto como limite superior do tempo esperado para a conclusão das transmissões na banda de controle. Devido ao fato de não considerar descarte por esgotamento do número de tentativas, a curva do modelo se distancia suavemente da curva da simulação quando o número de nós aumenta e começam a ocorrer colisões na rede. A quantidade percentual de pacotes entregues é mostrada na Figura 4(b).

\section{Resultados Obtidos}

Nesta seção, descreveremos o cenário de simulação utilizado para a comparação do desempenho dos dois protocolos de descoberta de vizinhança propostos com outro já existente na literatura, o MDND [Park et al. 2015]. Os protocolos foram analisados considerando a taxa de acertos primários, ou seja, a porcentagem dos ângulos obtidos através do mapa que estão corretos e permitem a pronta transmissão, e o tempo levado para a descoberta total da rede para cada protocolo.

O cenário reproduz uma sala 10 m x 10 m onde os nós são espalhados aleatoriamente com distribuição uniforme. Cada nó possui uma interface capaz de operar na banda 
de controle omnidirecional e outra que opera na banda de ondas milimétricas. Na banda de ondas milimétricas, as antenas direcionais são estáticas, ou seja, não se movem em torno do próprio eixo, em nenhuma das direções. As antenas são eletronicamente chaveáveis (Electronically Steerable). O modelo de antena adotado foi o Idealized Flat-Top Antenna System, que é amplamente explorado na literatura [An et al. 2011, Vasudevan et al. 2004].

Os experimentos foram realizados usando bibliotecas matemáticas da linguagem Python em cerca de 10000 simulações variando os parâmetros listados na Tabela 1. Ao considerar o número de feixes como variável, adotamos redes com 15 nós, quando o números de nós está variando, os nós permanecem com a configuração de 8 feixes. Os gráficos plotam as barras de erro relativas aos intervalos de confiança de $95 \%$.

\begin{tabular}{|c|c|}
\hline \multicolumn{2}{|c|}{ Parâmetros da Simulação dos Protocolos Multibanda } \\
\hline Cabeçalho das Camadas Física + MAC & 50 Bytes \\
\hline Ack & 31 Bytes \\
\hline Sector Sweep & 26 Bytes \\
\hline Sector Sweep Feedback & 31 Bytes \\
\hline Comprimento do quadro Mapa & (10*Número de Nós) Bytes \\
\hline Comprimento do quadro de Dados & 1024 Bytes \\
\hline RSV_REQ e RSV_RES & 20 Bytes \\
\hline SIFS & $10 \mu \mathrm{S}$ \\
\hline Slot & $9 \mu \mathrm{S}$ \\
\hline DIFS & $28 \mu \mathrm{S}$ \\
\hline SIFS (Ondas Milimétricas) & $3 \mu \mathrm{S}$ \\
\hline SBIFS & $1 \mu \mathrm{S}$ \\
\hline Ack Timeout (Banda de Controle) & $100 \mu \mathrm{S}$ \\
\hline Ack Timeout (Ondas Milimétricas) & $300 \mu \mathrm{S}$ \\
\hline Taxa de Transferência (Banda de Controle) & $6 \mathrm{Mbps}$ \\
\hline Taxa de Transferência (Ondas Milimétricas) & 25,8 Mbps \\
\hline Número de Nós & {$[5,10,15,20,25]$} \\
\hline Número de Feixes & {$[4,8,16,32]$} \\
\hline
\end{tabular}

\subsection{Taxa de Acertos Primários}

A taxa de acertos primários é a medida do número de sucessos que um nó obteve ao transmitir a partir das informações providas pelo nó líder no mapa. Essa métrica revela o quão precisa é a informação de localização dos nós obtida. Quanto mais acertos primários houverem, mais precisa foi a informação obtida.

Há um erro intrínseco à medida do ângulo causado pelo beamforming. Análogo à quantização, o processo de beamforming perde parte da informação do ângulo, posicionando um nó de forma relativa ao feixe encontrado durante o processo. Essa perda de informação insere erro nos cálculos, diminuindo o número de acertos primários. A Figura 5, mostra a taxa de acertos primários do protocolo MuNDi MaPP. Os demais protocolos não dependem da divulgação de mapas, portanto essa métrica não se aplica a esses protocołos. A Figura 5 mostra que há uma diferença em média de cerca de $20 \%$ no número de acertos 


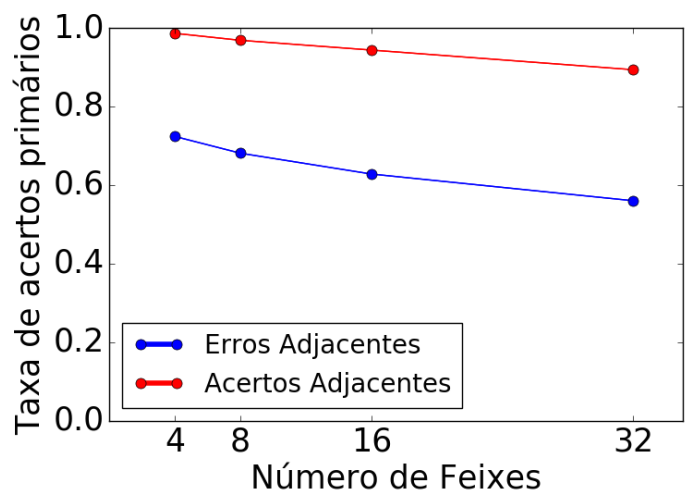

(a)

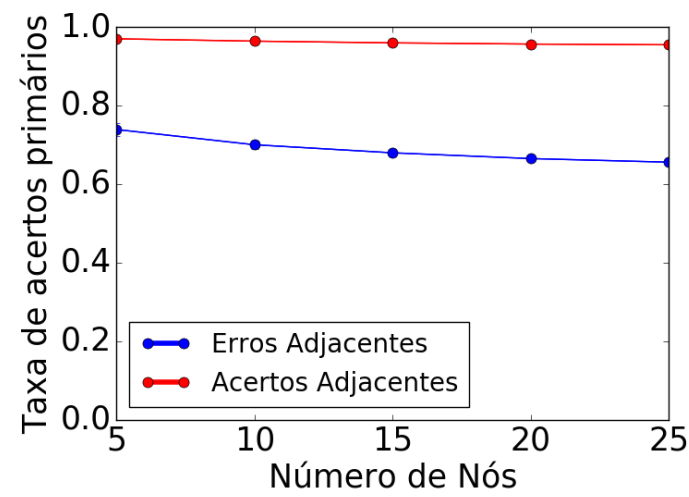

(b)

Figura 5. (a) Taxa de acertos primários quanto ao número de feixes; (b) Taxa de acertos primários em relação ao número de nós, comparando considerar ou não erros por 1 feixe

primários entre considerar ou não os feixes adjacentes. Assim, iniciar as buscas por um vizinho não encontrado pelos feixes adjacentes, pode diminuir o tempo em relação a uma busca sequencial. Outra alternativa é aumentar a largura dos feixes na recepção, desde que não resulte na perda total do enlace.

\subsection{Tempo de Descoberta da rede}

Para avaliar a eficiência dos mecanismos de descoberta, medimos também o tempo levado para que toda a rede seja descoberta, incluindo a troca de pacotes entre todos os nós da rede. Ao avaliar o protocolo MDND, consideramos que dado um par de nós, só é necessário realizar beamforming uma vez. Também consideramos que os nós só consultam o AP acerca de informações das capacidades da rede apenas uma vez. Para o protocolo MuNDi MaPP não adotamos um norte comum aos nós, o que implica na realização do beamforming nas duas direções do líder para o nó e vice-versa. não utilizamos o recurso de refinamento do beamforming, tanto para o MuNDi MaPP quanto para o Go MuNDi.

Com base no resultado exposto na Figura 6(a) podemos concluir que o protocolo MDND leva mais tempo para descoberta total da rede do que os demais. Isso se deve ao fato de este protocolo realizar mais acessos ao canal de controle em relação aos demais, o que em uma rede densa resulta em uma descoberta que leva mais tempo. A utilização do canal de controle, que possui taxa de transmissão menor e ainda a disputa pelo meio, ocasionou ao MDND em um cenário mais denso e que considera a descoberta de toda rede o uso de um intervalo maior para as suas descobertas. A Figura 6(b) também mostra que o protocolo Go MuNDi precisa de 22\% mais tempo que o protocolo MuNDi MaPP, devido às mensagens de feedback que ocasionam sucessivas disputas pelo meio.

Podemos observar também que ao considerar os feixes adjacentes, no que chamamos MaPP Modificado, o protocolo MuNDi MaPP, mais sensível ao aumento no número de feixes, pode ter seu desempenho melhorado, como nas Figuras 6(b) e (c). Esse resultado está de acordo com o discutido na Subseção 5.1, ou seja, ao considerar os feixes adjacentes ao feixe de recepção ou transmissão, pode-se melhorar o desempenho dos protocolos no que tange ao período até que a primeira transmissão de um enlace possa ser 
realizada, por outro lado, isto resulta em um enlace de qualidade menor.

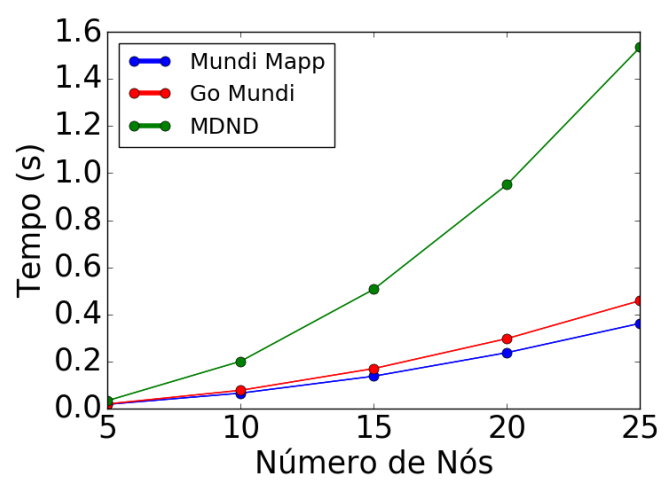

(a)

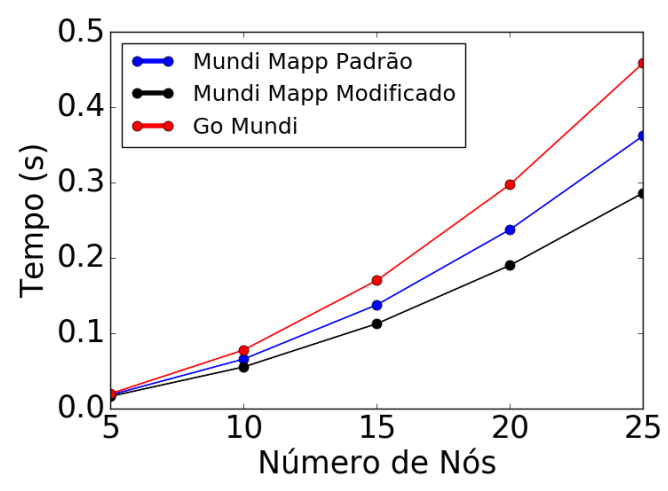

(b)

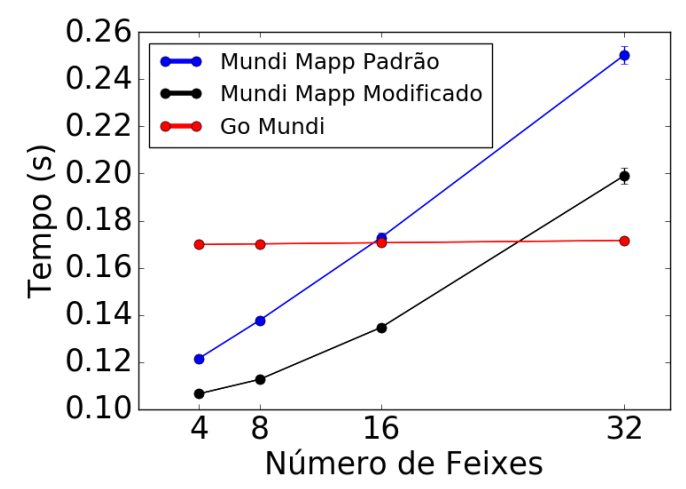

(c)

Figura 6. (a) Comparação entre os três protocolos MDND, Go MuNDi e MuNDi MaPP; (b) Detalhe do tempo de descoberta dos protocolos Go MuNDi e MuNDi MaPP quanto ao número de nós; (c) Detalhe do tempo de descoberta dos protocolos Go MuNDi e MuNDi MaPP quanto ao número de feixes

\section{Conclusões e Trabalhos Futuros}

As redes em ondas milimétricas impõem condições desafiadoras à descoberta de vizinhança. As informações de controle são críticas para a manutenção da rede e estão sujeitas a condições de propagação severas e à direcionalidade. Portanto, protocolos multibanda, como os aqui apresentados, são soluções viáveis para o tráfego de informação de controle, como a localização dos vizinhos. Dentre os protocolos apresentados, Go MuNDi e MuNDi MaPP, ressaltamos a possibilidade de descoberta global com eficiência em relação ao protocolo já existente na literatura MDND. Esses protocolos reduziram consideravelmente o tempo de descoberta de redes densas, partindo de um conhecimento com respeito à vizinhança e sua localização. Futuramente pretende-se expandir os protocolos para oferecerem suporte a múltiplos saltos, permitindo enlaces mais curtos e com maiores taxas de transferência.

\section{Referências}

An, X., Prasad, R. V., and Niemegeers, I. (2011). Impact of antenna pattern and link model on directional neighbor discovery in $60 \mathrm{ghz}$ networks. IEEE Transactions on Wireless Communications, 10(5):1435-1447. 
Cardoso, K. V. and de Rezende, J. F. (2012). Increasing throughput in dense 802.11 networks by automatic rate adaptation improvement. Wireless Networks, 18(1):95112.

Korakis, T., Jakllari, G., and Tassiulas, L. (2008). Cdr-mac: A protocol for full exploitation of directional antennas in ad hoc wireless networks. IEEE Transactions on Mobile Computing, 7(2):145-155.

Ning, J., Kim, T.-S., Krishnamurthy, S. V., and Cordeiro, C. (2011). Directional neighbor discovery in $60 \mathrm{ghz}$ indoor wireless networks. Performance Evaluation, 68(9):897915 .

Nitsche, T., Cordeiro, C., Flores, A. B., Knightly, E. W., Perahia, E., and Widmer, J. C. (2014). Ieee 802.11 ad: directional $60 \mathrm{ghz}$ communication for multi-gigabit-persecond wi-fi. IEEE Communications Magazine, 52(12):132-141.

Niu, Y., Li, Y., Jin, D., Su, L., and Vasilakos, A. V. (2015). A survey of millimeter wave communications (mmwave) for 5g: opportunities and challenges. Wireless Networks, 21(8):2657-2676.

Olivier, A., Bielsa, G., Tejado, I., Zorzi, M., Widmer, J., and Casari, P. (2016). Lightweight indoor localization for 60-ghz millimeter wave systems. In Sensing, Communication, and Networking (SECON), 2016 13th Annual IEEE International Conference on, pages $1-9$. IEEE.

Palacios, J., Casari, P., and Widmer, J. (2017). Jade: Zero-knowledge device localization and environment mapping for millimeter wave systems. In INFOCOM 2017-IEEE Conference on Computer Communications, IEEE, pages 1-9. IEEE.

Park, H., Kim, Y., Song, T., and Pack, S. (2015). Multiband directional neighbor discovery in self-organized mmwave ad hoc networks. IEEE Transactions on Vehicular Technology, 64(3):1143-1155.

Sakamoto, H. (1943). On the distributions of the product and the quotient of the independent and uniformly distributed random variables. Tohoku Mathematical Journal, First Series, 49:243-260.

Sim, G. H., Nitsche, T., and Widmer, J. C. (2016). Addressing mac layer inefficiency and deafness of ieee802. 11ad millimeter wave networks using a multi-band approach. In Personal, Indoor, and Mobile Radio Communications (PIMRC), 2016 IEEE 27th Annual International Symposium on, pages 1-6. IEEE.

Vasudevan, S., Kurose, J., and Towsley, D. (2004). On neighbor discovery in wireless networks with directional antennas. 